\title{
Effects of Shroud Curvature on the Performance of Centrifugal Compressor Blade
}

\author{
Kaiyuan $\mathrm{Hao}^{1, \mathrm{a}}$, Weimin Wang ${ }^{1, \mathrm{~b}}$, Yongqiang Shi ${ }^{1, \mathrm{c}}$ Shasha Wang ${ }^{1, \mathrm{~d}}$ \\ ${ }^{1}$ Diagnosis and Self-recovery Engineering Research Center, Beijing University of Chemical \\ Technology, Beijing 100029, China \\ ahaokaiyuan0309@163.com, bwwmbuct@163.com, 'shiyq1987@gmail.com, \\ dsusan202020@163.com
}

Keywords: centrifugal compressor, blade, stress, aerodynamic

\begin{abstract}
The purpose of the study described in this paper was to investigate the impact of shroud curvature on the performance of a centrifugal impeller or stage. The paper discusses a computational fluid dynamics (CFD) study done to assess the influence of shroud curvature on impeller performance. The computational fluid dynamics (CFD) and finite element analyses (FEA) methods were used to describe the various designs of the impeller.

Aerodynamic and mechanical analysis results are presented for four impellers of varying cover curvature and axial length. The aerodynamic results showed there were clear aerodynamic benefits to decreasing the curvature along the impeller shroud. The mechanical analytical results showed that the impeller with the lowest curvature or longest axial length provided the highest performance; it also yielded the lowest Von Mises stress level.

In closing, there are clear aerodynamic benefits to decreasing the curvature along the impeller shroud but these benefits must be weighed against the impact on the rotordynamic considerations Comments are offered regarding the rotordynamic issues that must be considered when increasing the length of impellers.
\end{abstract}

\section{Introduction}

Given the increasing costs for energy, process compressor users have demanded higher performance from centrifugal compressor OEMs. Nowhere is this truer than in the large-scale equipment used for liquefied natural gas, gas to liquid, ethylene or the like. These large trains often press the power limits of very high horsepower turbines. Therefore, it is imperative that the OEMs provide the maximum achievable efficiency in their aerodynamic designs.

Researchers have invested a lot of effect to improve the aerodynamic design of the centrifugal compressor. Many of these efforts focused on centrifugal impellers as they represent the most critical element of a centrifugal stage. Some researchers have achieved the improvements via arbitrary blade shapes, splitter-blade impellers and etc. Others have focused on the stationary components such as diffusers (vaned or vaneless); return channel, inlets, volutes, and sidestreams. A tremendous amount of work has been done on them; i, e., Hamid R Hazbyand emi Kim [1, 2] have studied the effects of diffuser and inlet volute on the overall performance of the compressor. However, it is still universally recognized that stage efficiency must begin with the impeller. The adjustment of stationary components can not overcome a poorly performing impeller.

Consider of the complexity, the heavy mole weight applications also require high flow coefficient impellers that operate at high inlet relative Mach numbers. Further, the compressors are typically multistage arrangements, having as many as 6 or 7 stages per casing. Therefore, rotor dynamics can become an problem if the staging is excessively long. Even further, high flow 
coefficient impellers normally followed with high stress levels that limit the allowable operating speeds. Sorokes $[3,4]$ have studied the design process for high inlet relative Mach number impellers. The research shows that shroud curvature has a significant impact on inlet relative Mach number.

The purpose of the study described in this paper was to investigate the impact of shroud curvature on the performance of a centrifugal impeller or stage. This issue seems simple just to increasing the curature radius but this is a serious problem to designers in the process centrifugal compressor industry. Increase the curature radius cause a low inlet relative Mach number and improve the performance. However, this will also increase the axial length of the impeller and bearing span. If the bearing span becomes excessive, rotordynamic issues could result. Therefore, the key is to find or understand the compromise between aerodynamics and rotor dynamics performance.

The paper investigates the aerodynamic, rotordynamic, and mechanical trend associated with varying the shroud curvature and axial length of a centrifugal impeller. Computational fluid dynamics (CFD) has been shown to be very reliable in the design and analysis of new or existing aerodynamic component designs. There are a vast number of references available on the use of CFD for stage and/or component design .Albert[5] and Filippo[6] have used CFD method analyzed the effect of the inlet flow separation and vaned diffuser on compressor performance.

CFD results are presented to show the achievable preformace benefits. Stress analyses are also used to show the potential mechanical considerations. Finally, the conclusions are offered on overall performance of the long impeller.

\section{Numerical Simulation}

CFD Model / Methods. The CFD computational domain consists of a rotating impeller, a vaned diffuser, followed by a return bend and return channel. The computational domain shown in Figure 1.All impeller blade passages are modeled in the present simulations for achieve a more accurate result.

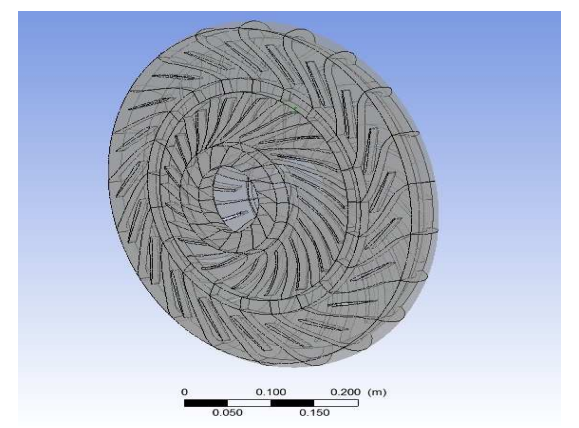

Fig. 1. Sketch map of the computational domain

All of the grids used in the analyses consisted of hexahedral elements. In all cases, the minimum face angle was greater than 20 degrees and the maximum face angle was less than 160 degrees. The frozen-rotor approach is adopted to consider the rotor/stator interface between a stationary and a rotating component.

All CFD analyses employed air treated as an ideal gas for a working fluid. The inflow boundary condition includes uniformly distributed total temperature and total pressure and a specified mass flow rate was used as an outlet boundary condition. All solid walls were modeled as hydraulically smooth with an adiabatic condition. The solutions were considered converged when the maximum values of all momentum and mass flow residuals dropped below a maximum value of $1.0 \mathrm{e}-5$. 
FEA Model / Methods. The finite element impeller models used in this study were including all blades models. The tetrahedral mesh was used in this study. These meshs have been found accurately enough to provide satisfactory results. All boundary nodes were rotated into the cylindrical coordinate system and coupled to their corresponding locations. An axial and tangential restraint was applied to the blade centerline node at the impeller inside diameter A rotational speed of $1200 \mathrm{r} / \mathrm{min}$ was applied as the loading condition. Likewise, the CFD result was applied as a pressure loading condition on the blade.

The analyses showed that as the axial length of the impeller increased the stress level of the impeller decreased. Depending on the mechanical design criteria, the model with the lowest overall VonMises nodal stress was being sought to determine the best impeller design.

\section{Analytical Results --Aerodynamics}

Having discussed the aerodynamic and mechanical analysis procedures, the discussion will now turn to the results obtained for the four impellers with different axial length. The meridional profiles for the four impellers are shown in Figure 2.

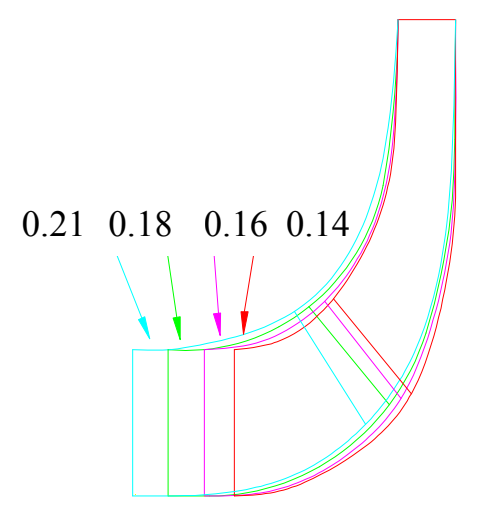

Fig. 2. Cross-Sections of different Impellers

Vector plots at the impeller exit plane for the 0.14 and the $0.21 \mathrm{Ax} / \mathrm{D} 2$ impellers are shown in Figure 3. The vectors are colored by relative Mach number and reflect the impeller exit conditions at the design flow rate. Velocity vector plots near the suction surface for each impeller, also at the design flow rate, are shown in Figure 4. Figures 3 and 4, one can see that the impeller exit velocities and/or relative Mach number are more uniform as a result of reduced shroud curvature. Note also the reduction in relative Mach number along the shroud between the $0.14 \mathrm{Ax} / \mathrm{D} 2$ and the 0.21 Ax/D2 impellers in Figure 4. The reduction in local curvature has reduced the Mach number in the impeller exit plane. While not obvious from the figures provided, the longer impeller did provide wider flow range than the shorter versions due in part to the lower Mach number. The additional range would be advantageous for many process compressor applications.

The downstream stationary flow path components also benefit from the improved impellers. The meridional projection of velocity throughout the analytical domain is given in Figure 5. This figure shows the improvement in the diffuser flow field as a result of the reduction in the impeller exit wake region. Note the absence of the low velocity region along the diffuser shroud surface in the $0.21 \mathrm{Ax} / \mathrm{D} 2$ design versus the $0.14 \mathrm{Ax} / \mathrm{D} 2$ stage. Recall that the reduction in the impeller exit wake exit was achieved through the reduction in shroud curvature. 

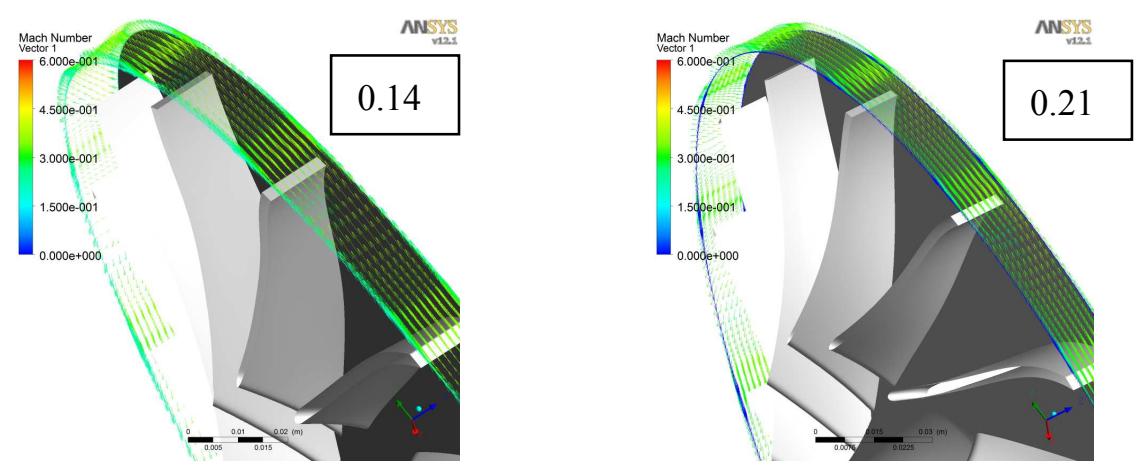

Fig. 3. Impeller Exit Velocity Vectors Colored by Relative Mach Number
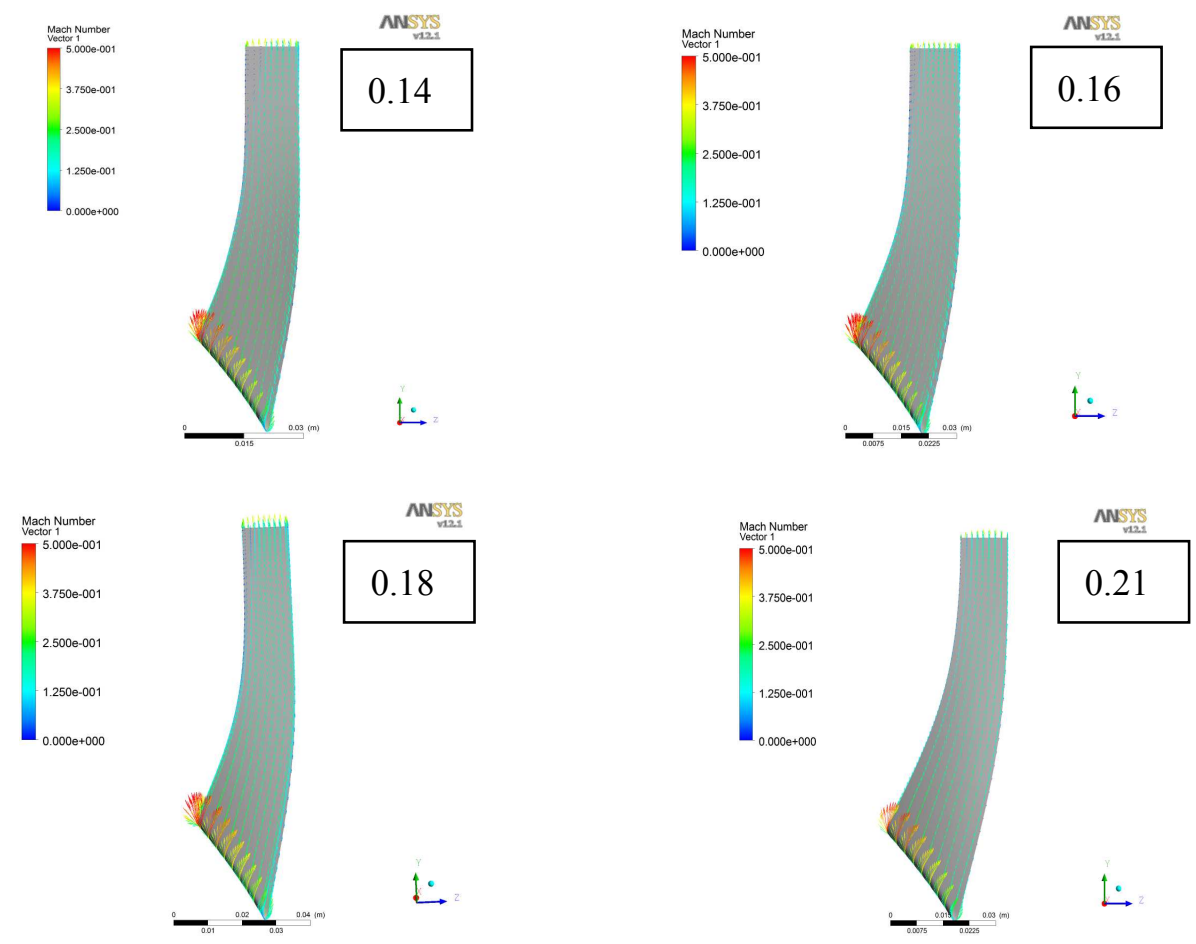

Fig. 4. Velocity Vectors Colored by Relative Mach Number-Near Suction Surface

Finally, the performance curves for all of the new impellers were given in Figure 6.Of the four, the $\mathrm{Ax} / \mathrm{D} 2=0.21$ stage shows the highest preformance and widest range. In other words, the aerodynamic performance of compressor stage is great increased with the reduction in shroud curvature.

\section{Analytical Results --Mechanical}

To study the mechanical properties of several impeller, the maximum values of several critical stresses were compared in Table 1 . The values in the table were normalized using the stresses for the $\mathrm{Ax} / \mathrm{D} 2=0.21$ impeller. That is, the stresses for the $\mathrm{Ax} / \mathrm{D} 2=0.21$ impeller are shown as $100 \%$ and the various stress levels for the other designs are shown as percentages of those for the Ax/D2=0.21 impeller. 


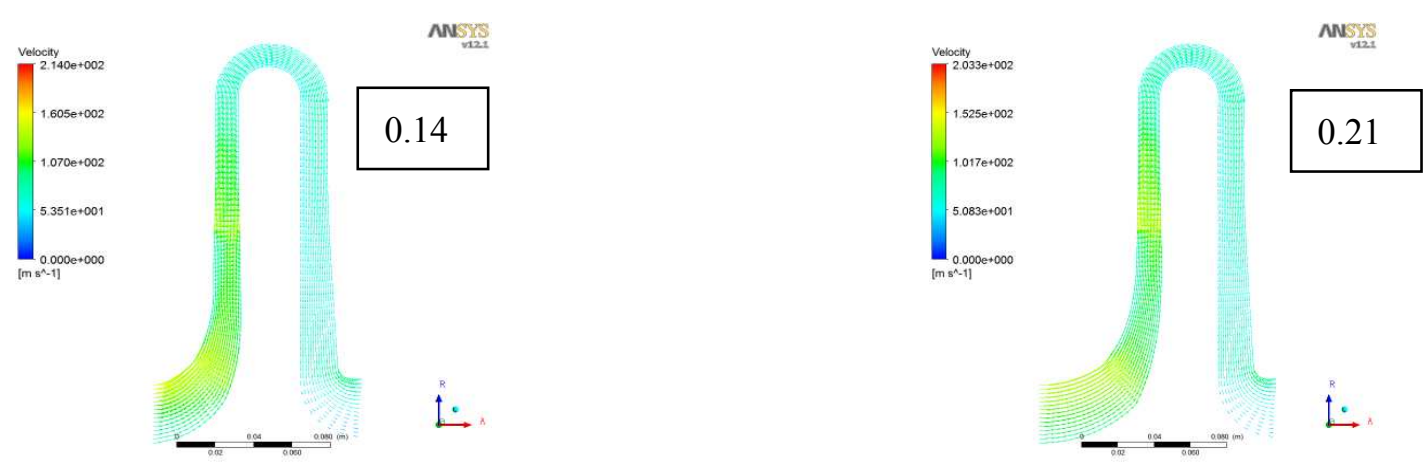

Fig. 5. Average Meridional Velocity Vector
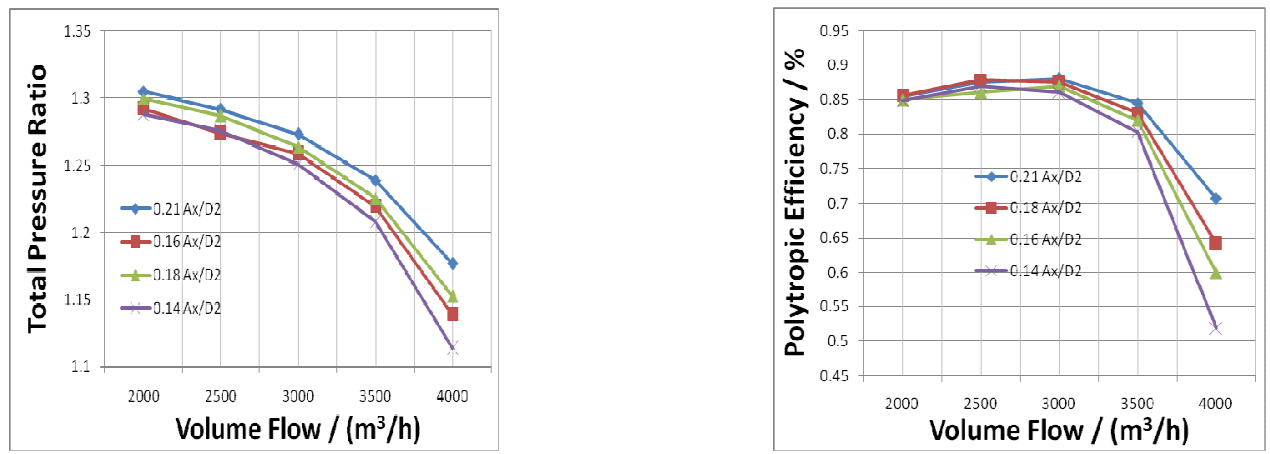

Fig. 6. The stage Performance curves of different design impeller

As can be seen, the stress levels in the longest impeller (i.e., Ax/D2 of 0.21) are, on average, the lowest while the highest stresses are found in the $0.14 \mathrm{Ax} / \mathrm{D} 2$ impeller. In particular, the OEM uses the Von Mises stress as a critical assessment parameter in comparing new designs and the maximum Von Mises stress is nearly $14.3 \%$ higher in the 0.14 Ax/D2 impeller than in the 0.21 ones. In short, while the impeller with the lowest curvature or longest axial length provided the highest performance, it also yielded the lowest Von Mises stress level. However, it does not means the length of impeller can be increased no limit. As noted earlier, the impeller with the lowest cover curvature $(\mathrm{Ax} / \mathrm{D} 2=0.21)$ has the longest axial length. This means that the bearing span for a collection of such impellers would be considerably longer than a collection of 0.14 Ax/D2 impellers. It is possible that the longer impellers and resulting longer stage axial length that was unacceptable from a rotor dynamics perspective. Furthermore, the longer impellers also will weigh more than their shorter ones. This increases the rotating mass and moment of inertia. In short, the potential benefits derived from the reduced curvature or longer length must be weighed against the possible rotordynamic consequences.

Table 1 The Relative Stress Levels of Impeller

\begin{tabular}{ccccc}
\hline $\mathrm{Ax} / \mathrm{D} 2$ & $\mathrm{Sn}$ & $\mathrm{St}$ & $\mathrm{SEQV}$ \\
\hline & 0.21 & $100.0 \%$ & $100.0 \%$ & $100.0 \%$ \\
0.18 & & $103.3 \%$ & $103.0 \%$ & $102.4 \%$ \\
0.16 & $103.6 \%$ & $99.52 \%$ & $102.6 \%$ \\
0.14 & $103.47 \%$ & $105.7 \%$ & $114.3 \%$ \\
\hline
\end{tabular}




\section{Conclusions}

In this study, the impact of shroud curvature on the performance of a centrifugal impeller or stage was investigated. Aerodynamic and mechanical analysis results were presented for four impellers of varying cover curvature and axial length, and key conclusions of the investigation are summarized as follows.

(1)The aerodynamic analytical results showed there are clear aerodynamic benefits to decreasing the curvature along the impeller shroud. Efficiency and working range were both improved.

(2)The mechanical analytical results showed that the lowest curvature or longest axial length provided the lowest Von Mises stress level. The maximum Von Mises stress is nearly $14.3 \%$ higher in the short impeller than in the long impeller.

(3)It is no doubt that decreasing the curvature along the impeller shroud will improve the performance of the stage but these benefits must be weighed against the impact on the rotordynamic considerations. If a reasonable compromise can be found between the aerodynamic, stress, and rotordynamic, a superior design can be derived.

\section{Nomenclature}

$A x=$ axial length from shroud leading edge to hub plane at impeller exit

D2 = impeller exit diameter

$\mathrm{Sn}=$ Normal stress

$\mathrm{St}=$ Tangential stress

$\mathrm{SEQV}=$ maximum Von Mises nodal stress

\section{Acknowledgments}

This work was supported by the general Program of Natural Science Foundation of China (50975018).

\section{References}

[1] Hamid R Hazby., Liping Xu., Matthias Schleer ,Study of The Flow In A Vaneless Diffuser At Part Speed Operating Conditions,ASME .(2010) 1-11.

[2] Semi Kim, Junyoung Park, Kukyoung Ahn, Jehyun Baek, Improvement of the Performance of a Centrifugal Compressor by Modifying the Volute Inlet, J. Journal of Fluids Engineering 132(2010) 091101-1-091101-7.

[3] Sorokes, J.M., The Practical Application of CFD in the Design of Industrial Centrifugal Impellers, Texas A\&M Turbomachinery Symposium Proceedings. (1993).

[4] Sorokes,J.M.,Kopko,J.A,High Inlet Relative Mach Number Centrifugal Compressor Impeller Design, ASME. (2007) GT2007-27864.

[5] Albert Kammerer,Reza S., Abhari.Unsteady Computational Fluid Dynamics Investigation on Inlet Distortion in a Centrifugal Compressor,J.Journal of Turbomachinery. 132 (2010) 1-9.

[6] Michele Marconcini,Filippo Rubechini, Andrea Arnone, Seiichi lbaraki,Numerical Analysis of the Vaned Diffuser of a Transonic Centrifugal Compressor, J.Journal of Turbomachinery. 132(2010)1-7. 